\title{
Fractionation of Fab glycosylated immunoglobulin G with concanavalin A chromatography unveils new structural properties of the molecule
}

\author{
Tao Huang ${ }^{1, *}$, Xueling Chen ${ }^{1, *}$, Huan Gu${ }^{1}$, Conghui Zhao ${ }^{2,3}$, Xingmu Liu ${ }^{1,4}$, Meiling \\ Yan ${ }^{1}$, Xiaodong Deng ${ }^{1}$, Zaiping Zhang ${ }^{1}$, Jiang Gu ${ }^{1,3}$ \\ ${ }^{1}$ Department of Pathology and Provincial Key Laboratory of Infectious Diseases and Immunopathology, Collaborative and \\ Creative Center, Molecular Diagnosis and Personalized Medicine, Shantou University Medical College, Shantou, Guangdong, \\ 515041, China \\ ${ }^{2}$ Department of Oral Pathology, Beijing Stomatological Hospital, Capital Medical University, Beijing, 100050, China \\ ${ }^{3}$ Department of Pathology, Beijing University Health Science Center, Beijing, 100083, China \\ ${ }^{4}$ Department of General Surgery, Second Affiliated Hospital, Shantou University Medical College, Shantou, Guangdong, \\ 515041, China \\ *These authors contributed equally to this work
}

Correspondence to: Jiang Gu, e-mail: 2523381625@qq.com

Keywords: IgG, glycosylation, ConA, asymmetry, symmetry

Received: February 25, $2016 \quad$ Accepted: April 11, 2016

Published: April 28, 2016

\section{ABSTRACT}

Concanavalin A (ConA) chromatography has been extensively used to separate asymmetric Immunoglobulin G (IgG), which possesses oligosaccharide attached to one of the two $F\left(a b^{\prime}\right)_{2}$ arms, from symmetric IgG with no glycan attached to Fab fragments. In this study, applying affinity chromatography, silver stain, Western blot and lectin stain techniques, $\mathrm{N}$ - linked oligosaccharide attached to Fab fragment was demonstrated to be exposed on the surface of the protein and be accessible by ConA. In contrast, $\mathbf{N}$ - linked oligosaccharide attached to asparagine (Asn) 297 of IgG Fc was located in the inside of the natural protein and was inaccessible by ConA. In addition to asymmetric IgG, there are also detectable level of IgG with both $F\left(a b^{\prime}\right)_{2}$ arms glycosylated that has not been reported previously. The discoveries of new basic molecular structure of IgG would have implications in understanding the function and properties of this important immune molecule with clinical applications.

\section{INTRODUCTION}

IgG has long been known as a glycoprotein with two highly conserved glycosylation sites located at Asn 297 in the $\mathrm{C}_{\mathrm{H}} 2$ domains of the Fe region. Further oligosaccharides may be found attached to the variable regions of $\operatorname{IgG}$ molecules [1]. The molecular structure of IgG is shown schematically in Figure $1 \mathrm{~A}$. The Fc glycosylation has been extensively studied to illustrate glycans' structure, influence on antibodies' function and glycoform changes associated with diseases. The common characteristic of $\operatorname{IgG~Fc}$ oligosaccharides is of the complex type with a constant heptasaccharide core. Additional sugar residues such as core fucose (Fuc), bisecting $\mathrm{N}$-acetylglucosamine (GlcNAc), galactose $(\mathrm{s})(\mathrm{Gal})$, and $\mathrm{N}$-aetylneuraminic acid(s) (NeuAc) may attached to the "core" [2] (Figure 1B).
In addition to Asn 297 of $\mathrm{Fc}$ fragment, 15-20\% of natural human IgG molecules bear N-linked oligosaccharides within the Fab fragments [3-5]. There are no glycosylation sites within the constant domains of either the light chain ( $\kappa$ - or $\lambda$ - chain) or the $\mathrm{C}_{\mathrm{H}} 1$ domain of heavy chains. Therefore, the oligosaccharides attach to the variable regions of the light chain $\left(\mathrm{V}_{\mathrm{k}}\right.$ or $\left.\mathrm{V}_{\lambda}\right)$, heavy chain $\left(\mathrm{V}_{\mathrm{H}}\right)$ or probably both. Attachment of the carbohydrates to the variable regions of light chains have been found in IgG from patients with multiple myeloma and an antiovomucoid monoclonal antibody (MAb-OM21) $[6,7]$. The glycans attach to the heavy chain variable regions of the antibody specific for $\alpha(1 \rightarrow 6)$ dextran (TKC3.2.2) and its effects on antigen-binding affinity have been evaluated $[8,9]$. The glycoforms attached to the Fab regions are of complex type with higher levels of galactorsylation and sialylation than those of Fc glycans $[10,11]$. 
Lectins are useful tools for fractionation and structural analysis of oligosaccharides and glycoproteins. ConA has long been used for separation of symmetric and asymmetric IgG molecules [12]. The symmetric IgG was thought to be Fab non-glycosylation fraction and the asymmetric IgG was the fraction with additional carbohydrate residue present in only one heavy chain variable region of two $\mathrm{F}(\mathrm{ab})_{2}$ arms. ConA is a tetrameric metalloprotein and specifically binds molecules containing the structure of sugar residue shown in Figure 1C [13]. Consequently, sugar residues which occur at non-reducing termini of sugar chains such as glucose $\alpha 1 \rightarrow$, Man $\alpha \rightarrow$ and GlcNAc $\alpha 1 \rightarrow$ residues and sugar residues which occur within sugar chains such as $\rightarrow 2$ glucose $\alpha 1 \rightarrow$ and $\rightarrow 2 \mathrm{Man} \alpha 1 \rightarrow$ residues can bind to this lectin. The $\rightarrow \beta 2$ Man $\alpha 1 \rightarrow$ residue conserved located in heptasaccharide core of the complex type oligosaccharides, therefore, by theory, all IgG molecules ought to react to ConA because of N-glycans attached to Asn 297 of IgG Fc. In reality, however, only $12 \%$ of natural human IgG bound to ConA column [12] with reasons unknown. This study resolved this paradox and unveiled that ConA was bound to Nlinked oligosaccharides attached to Fab regions that were exposed on the surface of IgG and were accessible by
ConA. In contrast, $\mathrm{N}$ - linked oligosaccharides atattached to Asn 297 of IgG Fc were located in the inside of the natural protein and were inaccessible by ConA. In addition, we further demonstrated that not all Fab glycosylated IgGs were asymmetric. Fab symmetrically glycosylated IgG, of which both arms of $\mathrm{F}\left(\mathrm{ab} \mathrm{b}_{2}\right)_{2}$ fraction were glycosylated, was successfully fractioned with ConA chromatography. This study does not only establish an effective method to fractionate Fab glycosylated IgG into three forms, i.e. Fab non-glycosylation IgG, Fab asymmetrically glycosylated IgG and Fab symmetrically glycosylated IgG with ConA affinity chromatography, but also unveil new facts about the basic structure and function of IgG molecule with theoretical and practical implications.

\section{RESULTS}

\section{The molecular characteristic of ConA+ IgG}

Human IgG was purified from serum with Protein G column and then fractionated with ConA Sepharose 4B. The difference in molecular weight of ConA+ and ConAIgG was examined with electrophoresis and silver stain (Figure 2A). Additional higher molecular weight bands
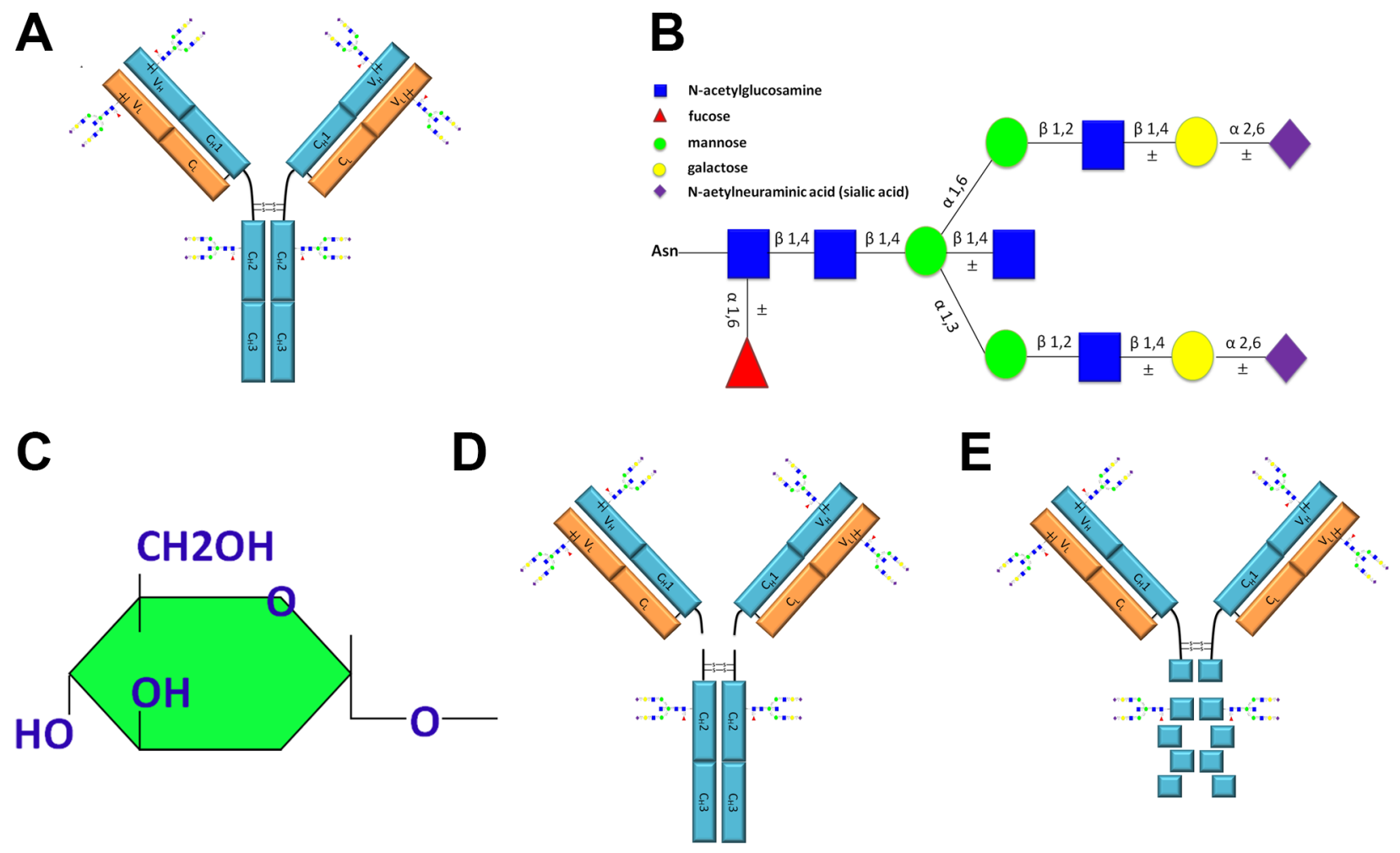

Figure 1: Schematic representation of IgG molecular structure including the glycoforms attached to IgG, the sugar residue that is recognizable by ConA and fragments of IgG obtained with enzyme digestion. (A) The molecular structure of $\mathrm{IgG}$ and potential glycosylation sites. The $\mathrm{IgG} \mathrm{Fc} \mathrm{C}_{\mathrm{H}} 2$ domains are conserved and are glycosylated through attachment of oligosaccharides at Asn 297. In addition to this conserved glycosylation site, extra oligosaccharides may be attached to the variable regions of the light chain, the heavy chain or both. (B) Schematic representation of the glycoforms attached to IgG. (C) The structure of sugar residue that can be specifically recognized by ConA. (D) Fab and Fc fragments derived from IgG with papain digestion. (E) F(ab') 2 and degraded Fc fragments obtained from IgG with pepsin digestion. \pm , represents possible glycosylation sites or additional sugar residues. 
existed above the $50 \mathrm{kDa}$ heavy chain band and the $25 \mathrm{kDa}$ light chain band in ConA+ IgG fraction and these two bands returned to the normal sizes following treatment with deglycosylation enzyme PNGase F. This indicates that the two higher molecular weight bands might be heavy chain and light chain with extra $\mathrm{N}$-glycans attaching to them. In order to determine the identity of these higher molecular weight bands, Western blot was performed with anti-Ig $\gamma$ Fc region, anti-Ig $\kappa$ light chain and anti-Ig $\lambda$ light chain antibodies. As shown in Figure 2B, the higher molecular weight monomer above the $50 \mathrm{kDa}$ heavy chain indeed was IgG heavy chain that can be identified with anti-Ig $\gamma$ Fc region antibody. Figure 2C, Figure 2D and Table 1 show that the higher molecular weight monomer above the $25 \mathrm{kDa}$ light chain was Ig $\kappa$ light chain. There are carbohydrates attached to the $\lambda$ light chain of human $\gamma \mathrm{G} 1$ myeloma proteins from the urine of patients with multiple myeloma has been reported [14]. However, no detectable level of N-glycans attached to $\lambda$ light chain of natural human $\operatorname{IgG}$ was found in this study.

\section{Oligosaccharides attached to Asn 297 are located in the inside of the molecule in its natural state}

The location of oligosaccharides of $\mathrm{IgG}$ was examined with affinity chromatography, silver stain, Western blot and lectin stain techniques. As shown in Figure $2 \mathrm{E}-2 \mathrm{~F}$, after denaturation and reduction with SDS and $\beta$-mercaptoethanol both ConA- and ConA+ $\mathrm{IgG}$ reacted with ConA indicating that the oligosaccharides attached to Asn 297 of Fc should be in the inside of the 3D protein structure and shielded from ConA. The reaction of $\mathrm{IgG}$ Fc fragments with ConA was also observed with lectin stain after papain digestion (Figure 3E). Under natural state, there was no reaction between ConA and Fc no matter if the Fc was derived from $\mathrm{IgG}$, ConA+ IgG or ConA- IgG (Figure 4A-4C). On the contrary, all IgG heavy chains bound to ConA after denaturation and reduction (Figure 5A-5B).

\section{Oligosaccharides attached to Fab fragments are responsible for the binding of IgG to ConA}

In electrophoresis, ConA+ IgG Fab fraction generates a band with higher molecular weight that would fall back to the normal molecular weight following treatment with deglycosylation enzyme PNGase F. In contrast, no molecular weight difference was detected between ConA+ and ConA- IgG Fc fragments (Figure 3A). Most of the higher molecular weight band in ConA+ IgG Fab fraction was Fd fragment, and a relatively small percentage was $\kappa$ light chain (Figure 3B-3D).

As shown in Figure $3 \mathrm{E}$, no ConA reactive Fab fragment was detected in ConA- IgG Fab fraction suggesting that all Fab glycosylated IgG molecules were collected into ConA+ IgG fraction. This further suggests that oligosaccharides attached to Fab fragments are likely exposed on the surface of the protein and can be bound by ConA. In addition, sialylation of N-linked oligosaccharides attached to IgG Fab and IgG Fc fragments both could be detected with SNA stain (Figure 3F).

\section{Effective fractionation of IgG with different degrees of Fab glycosylation}

An IgG molecule is composed of two identical light chains and two identical heavy chains, and form a structure with twofold symmetry [15]. When one of two $\mathrm{F}(\mathrm{ab})_{2}$, arms is glycosylated it is named asymmetry IgG [12]. The explanation for this phenomenon is that the competitive reactions of posttranslational modification and protein folding may result in the glycosylation of only a portion of the variable region [16]. In this study, we attempted to ascertain if there exist a form of Fab symmetrically glycosylated IgG of which both F(ab') arms are glycosylated. To clarify this supposition, we loaded $10 \mathrm{mg} \mathrm{IgG}$ onto ConA column, eluted with different solutions, collected the eluent at $0.5 \mathrm{~mL}$ per fraction and then determined the concentrations with absorbance at $A_{280}$. As shown in Figure 6A, three main fractions of $\mathrm{IgG}$ were isolated. The characteristics of these three fractions are shown in Figure 6B-6D. No molecular weight decline of ConA- IgG F(ab') fraction after PNGase $\mathrm{F}$ digestion indicates non-glycosylation of the $\mathrm{F}\left(\mathrm{ab}{ }^{\prime}\right)_{2}$ fragment (Figure 6B). A decline of one half of Fd' fragments of ConA + IgG F(ab') fraction in molecular weight after PNGase $\mathrm{F}$ digestion suggests the existence of Fab asymmetrically glycosylated IgG (Figure 6C). A decline of all Fd' fragments of ConA++ IgG F(ab') fraction in molecular weight after PNGase $\mathrm{F}$ digestion implies the existence of Fab symmetrically glycosylated IgG (Figure 6D).

\section{DISCUSSION}

Immobilized lectin columns have been used extensively for the fractionation and structural analysis of oligosaccharides and glycopeptides [13, 17]. For example, SNA specifically recognizes the Neu5Ac( $\alpha 2-6)$ Gal/GalNAc sequence [18]. MAA has affinity to oligosaccharides containing terminal sialic acid ( $\alpha 2-3)$ linked to $\mathrm{Gal} /$ GalNAc [19]. The carbohydrate binding property of GNA is terminally linked to mannose specifically [20]. DSA recognizes Gal- $\beta(1-4)-G l c N A c$ without terminal sialic acids [21]. It was demonstrated that unmodified hydroxyl groups at the C-3, C-4, and C-6 positions of a glycopyranosyl residue are essential for the interaction of ConA [22]. IgG molecules are glycoproteins containing at least N-linked carbohydrates in their Fc fragments [23]. The structure of oligosaccharides attached to Asn 297 of IgG Fc fragments is largely conserved, and in theory, the $\rightarrow \beta 2 \mathrm{Man} \alpha 1 \rightarrow$ residue located in heptasaccharide core of 
Table 1: The band of arrow points to (correspond to Figure 2) was determined with MALDI TOF MS analysis

\begin{tabular}{|l|l|l|}
\hline In-gel digestion & Protein hits & Mascot score \\
\hline \multirow{2}{*}{ The band of arrow points to } & Ig kappa chain C region & 56 \\
\cline { 2 - 3 } & Ig kappa chain V-IV region & 41 \\
\hline
\end{tabular}
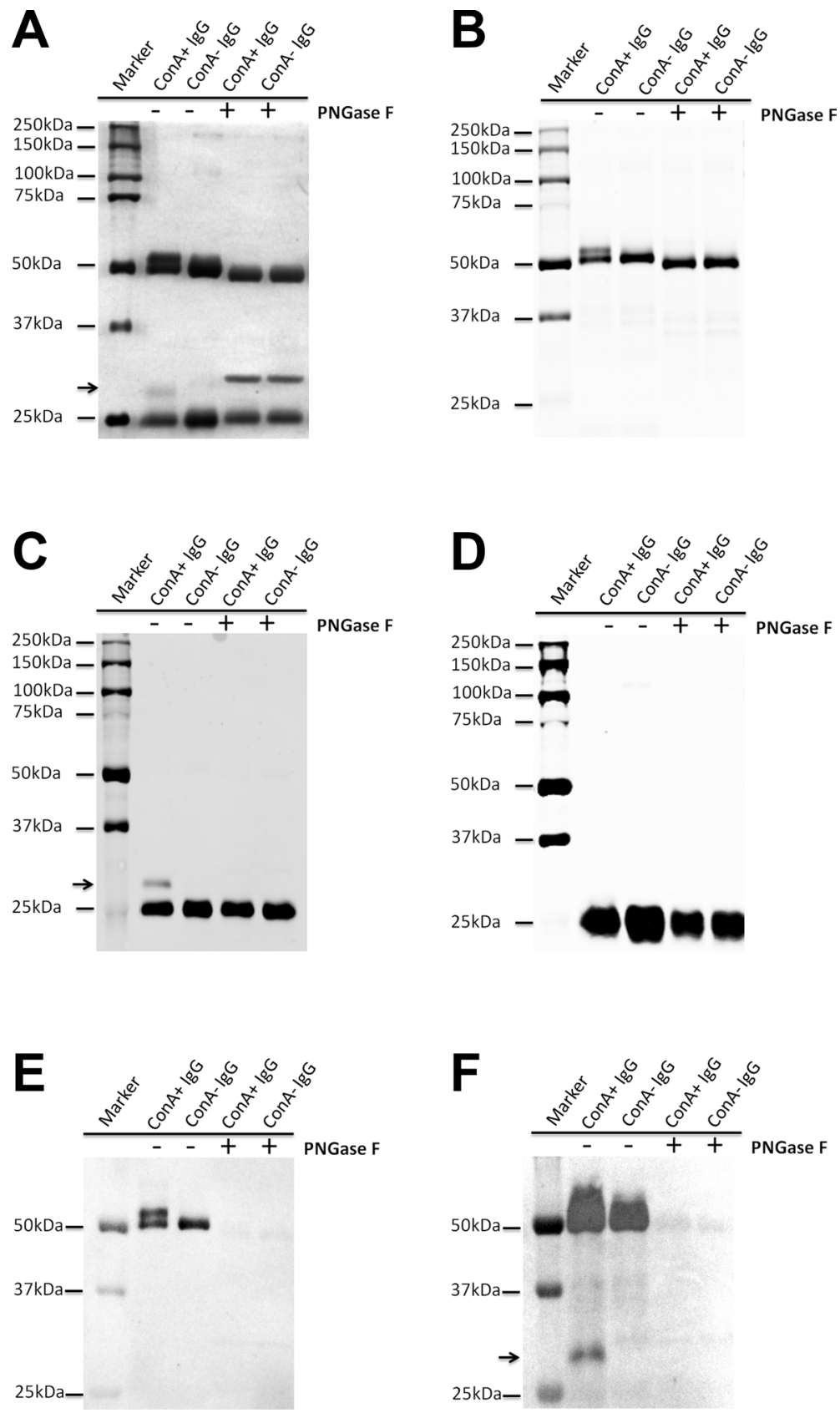

Figure 2: Extra N-glycans attach to part heavy chain or kappa light chain of ConA+ IgG. (A) Silver stain shows that there are higher molecular weight bands above the $50 \mathrm{kDa}$ heavy chain and the $25 \mathrm{kDa}$ light chain (arrow), which return to the normal sizes following treatment with deglycosylation enzyme PNGase F. (B-D) Western blot established that the higher molecular weight band above the $50 \mathrm{kDa}$ heavy chain was Ig $\gamma$ heavy chain (B), the higher molecular weight band above the $25 \mathrm{kDa}$ (arrow) was kappa light chain (C), but not lambda light chain (D). (E and F) Lectin stain shows that both heavy chain of ConA+ and ConA- IgG under denaturation and reduction conditions could react with ConA. Neither kappa nor lambda light chain could react with ConA except the higher molecular weight band above the $25 \mathrm{kDa}$ (arrow). The difference between $\mathrm{E}$ and $\mathrm{F}$ is in sample quantity, $\mathrm{E}(0.5 \mu \mathrm{g} / \mathrm{well})$ and $\mathrm{F}$ (1.5 ug/well). The sample quantity of A-D was $1 \mathrm{ug} /$ well. 
the biantennary complex type oligosaccharides should be recognized by ConA (Figure 1B). In reality, however, only $12 \%$ of natural human IgG bound to ConA column [12]. The present study resolved this paradox, we demonstrated that the Fc-linked oligosaccharides are located in the inside of the natural protein and are inaccessible by ConA. In contrast, oligosaccharides attached to Fab fragments are exposed on the surface of the protein and contribute to the binding with ConA column.

It has been known for years, based on investigation with crystallography, that oligosaccharides attached to Asn 297 of IgG Fc fragments are largely located internally
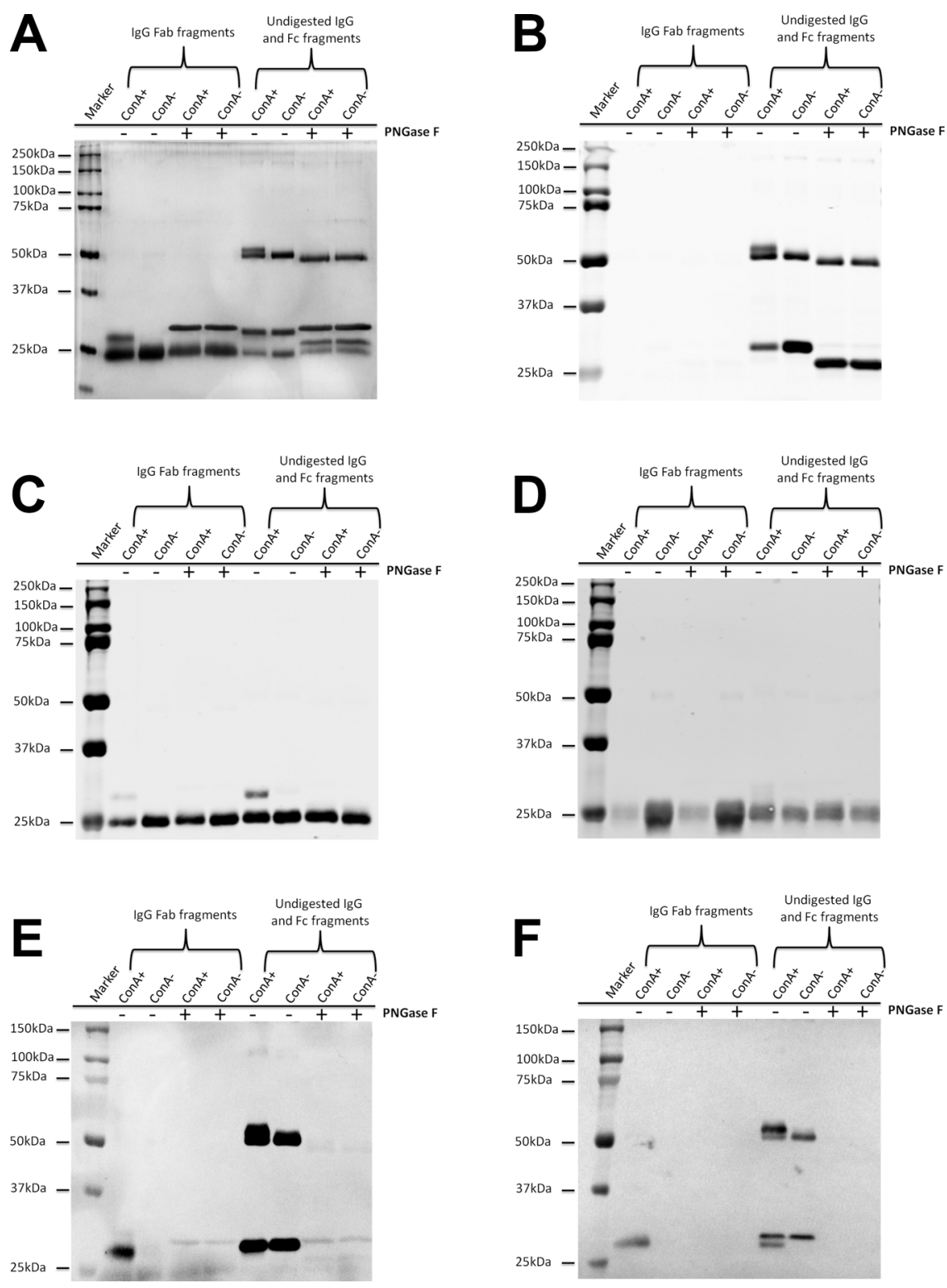

Figure 3: Extra N-glycans exist in Fab fragments but not in Fc fragments of ConA+ IgG. (A) Silver stain shows extra higher molecular weight band above the denatured and reduced Fab fragments but not above the denatured and reduced Fc fragments of ConA+ IgG. The higher molecular weight band returned to the normal size following treatment with deglycosylation enzyme PNGase F. (B-D) Western blot established that the higher molecular weight band above the denatured and reduced Fab fragments was a mixture of IgG heavy chain Fab fraction but not Fc fraction (B) and kappa light chain (C) but not lambda light chain (D). (E) ConA stain shows that both the higher molecular weight Fab fragments and the Fc fragments of ConA+ and ConA- IgG under denaturation and reduction conditions could react with ConA. (F) SNA stain shows that sialic acids are $\alpha-2,6-$ linked to oligosaccharides in both Fab fragments and Fc fragments. 
inaccessible by ConA regardless of the glycoforms. The accessibility of other lectins to Fc glycans remain a subject of farther investigation.

A kinetic explanation was proposed for the occurrence of Fab asymmetric glycosylation. Protein folding and posttranslational modification are the competitive reactions may result in only one arm of of $\mathrm{F}(\mathrm{ab})_{2}$ fraction being glycosylated [16]. Likewise, there might be Fab symmetric glycosylation IgG with both $\mathrm{F}\left(\mathrm{ab}{ }^{\prime}\right)_{2}$ arms being glycosylated. In this study, we demonstrated the existence of such IgG portion which was eluted with Regeneration Buffer $\mathrm{pH}$ 4.5. This experiment data not only verify the kinetic explanation of Fab glycosylation but also offers a technique to fractionate Fab asymmetrically glycosylated $\mathrm{IgG}$ and Fab symmetrically glycosylated IgG effectively.

The structure of Fc glycoforms, their influence on $\operatorname{IgG}$ function and their association to diseases have been studied extensively. Higher levels of sialylated Fc glycans were associated with reduced ADCC activity because of lower affinity binding to FcyRIIIa and/ or lower affinity binding to cell surface antigens [26]. Sialylation of the N-linked glycan of the IgG Fc fragment completely determined the anti-inflammatory activity of IgG $[27,28]$. The ADCC was enhanced by removing Fuc (fucose) from complex type oligosaccharides [29-32]. The GlcNAc of oligosaccharides in the Fc region binding to the collagenous lectin mannose-binding protein (MBP)
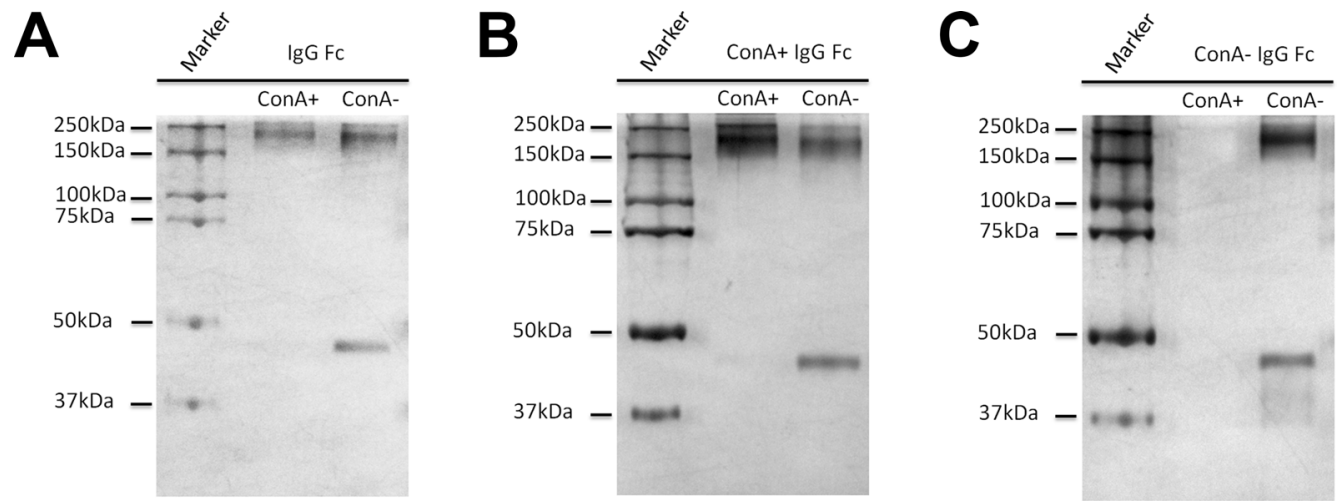

Figure 4: Oligosaccharides attached to Asn 297 of IgG Fc fragments are located in the inside of the natural protein of IgG and are inaccessible by ConA. Fc fragments (contain undigested $\operatorname{IgG}$ ) obtained from un-fractionated $\operatorname{IgG}(\mathbf{A}), \mathrm{ConA}+\operatorname{IgG}(\mathbf{B})$ and ConA- IgG $(\mathbf{C})$ were loaded onto ConA column. The bound and unbound fractions were collected and then examined with silver stain. The Fc fragments under non-reducing condition, regardless if they were obtained from un-fractionated $\operatorname{IgG}(\mathrm{A}), \mathrm{ConA}+\operatorname{IgG}(\mathrm{B})$ or ConA$\operatorname{IgG}(\mathrm{C})$, could not react with ConA indicating that oligosaccharides attached to Asn 297 were in the inside of the natural protein structure of IgG and were inaccessible by ConA.
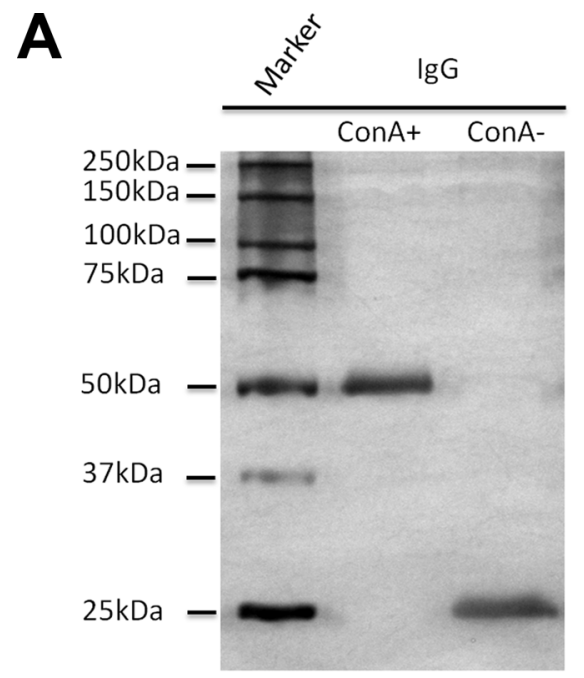

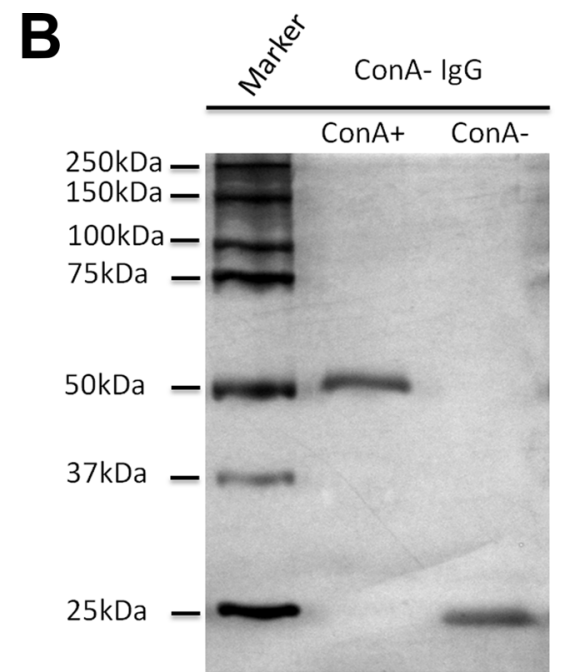

Figure 5: All Igy heavy chains bind to ConA column following denaturation and reduction with SDS and $\boldsymbol{\beta}$-mercaptoethanol. IgG (A) and ConA- IgG (B) were loaded onto ConA chromatography after denaturation and reduction, and then examined with silver stain. The heavy chain of $\operatorname{IgG}$ (A) including ConA- IgG (B) could bind with ConA due to glycans attached to Asn 297 that were exposed after denaturation and reduction. The $25 \mathrm{kDa}$ band of light chain could not bind to ConA column as no oligosaccharide was attached to it. 
contribute to a new mode of interaction of $\mathrm{IgG}$ with complement [33]. The changes in glycosylation patterns have also been found to be associated with diseases and aging [34-39]. Nevertheless, it should be noted that recent researches indicated that the sialylation $\mathrm{IgG}$ obtained by SNA fractionation with anti-inflammatory activity was essentially mediated by Fab not by Fc sialylation $[25,40]$. Combining with our current findings, we have reason to believe that some of previous conclusions about the function of Fc glycans, especially those established on the fractionation of IgG with lectin column, should be reevaluated due to the intramolecular location of Fc glycans.
Further investigations would be necessary to determine whether certain Fc glycan functions reported previously are in fact mediated by Fab glycosylation.

Fewer studies have been done to study IgG Fab glycans comparing with Fc glycans. The Fab glycosylation is presumed to be present on the variable region of heavy or light chain due to neither the light chain nor the heavy chain $\mathrm{C}_{\mathrm{H}} 1$ region contains a carbohydrate binding sequence (Asn-X-Ser/Thr; where $\mathrm{X}$ can be any amino acid except proline) [1, 41]. In a series of early studies, carbohydrate addition sites were found located in the variable region of myeloma, Bence-Jones and amyloid
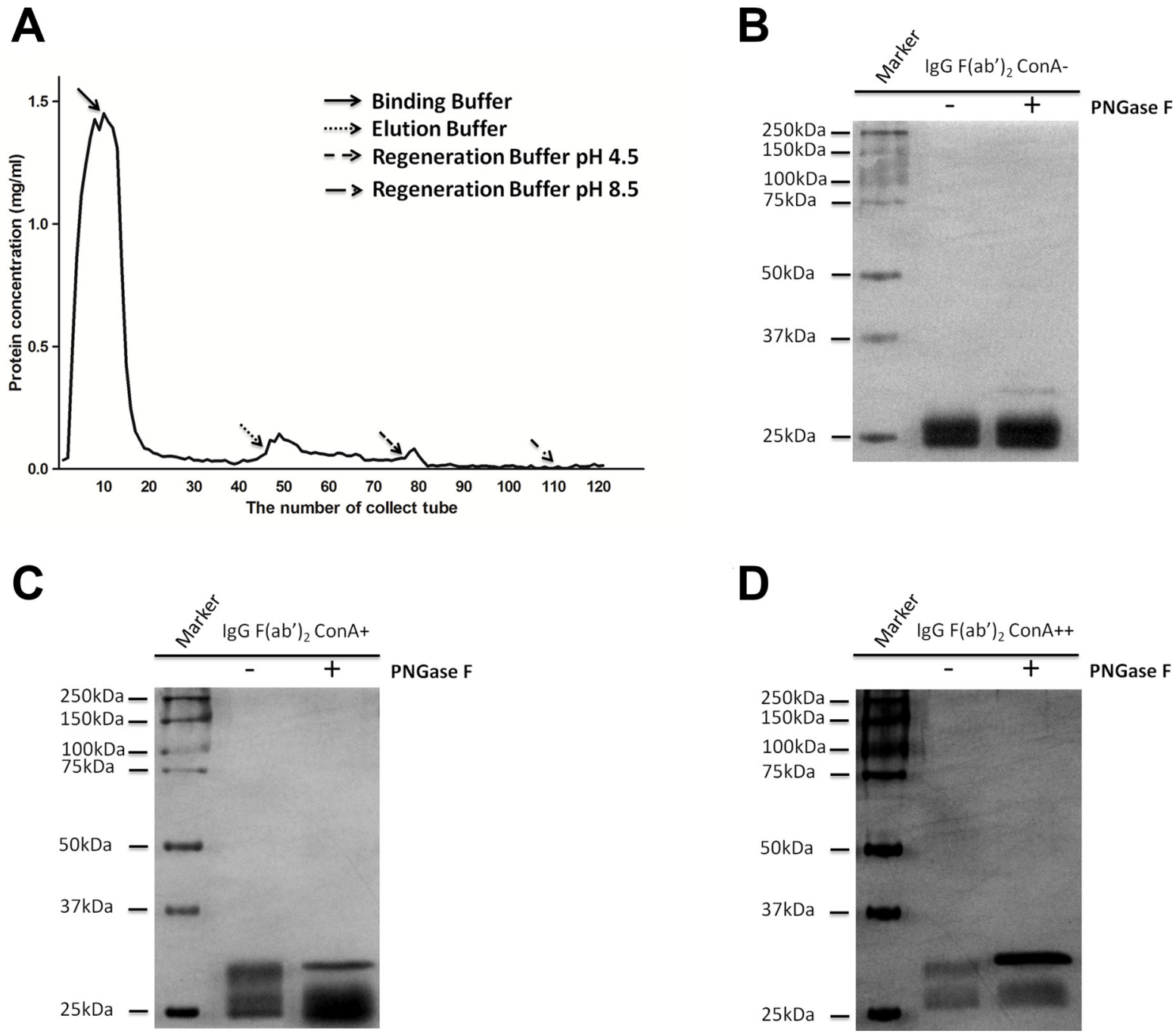

Figure 6: IgG was fractionated into three fractions: Fab non-glycosylation IgG (ConA- IgG), Fab asymmetrically glycosylated IgG (ConA+ IgG) and Fab symmetrically glycosylated IgG (ConA++ IgG) with ConA affinity chromatography. (A) Three fractions of IgG were isolated by eluting ConA column with different solutions including ConA- IgG eluted with Binding Buffer, ConA+ IgG eluted with Elution Buffer and ConA++ IgG eluted with Regeneration Buffer pH 4.5. (B-D) F(ab') fragment of IgG was loaded onto ConA column, and its three fractions were examined with silver stain with or without PNGase F digestion. (B) No molecular weight decline was observed for ConA- $\mathrm{F}\left(\mathrm{ab}^{\prime}\right)_{2}$ fraction after PNGase F digestion and this suggests that no N-glycans were attached to $\mathrm{F}\left(\mathrm{ab}\right.$ ') ${ }_{2}$ fraction. (C) When only half of Fd' fragments of ConA+ $\mathrm{F}\left(\mathrm{ab}\right.$ ') ${ }_{2}$ fraction were found to decline in molecular weight after PNGase F digestion, it was suggested that N-glycans were attached to F(ab') , fraction in only one arm asymmetrically. (D) When all Fd' fragments of ConA++ $\left.\mathrm{F}(\mathrm{ab})_{2}\right)_{2}$ fraction declined in molecular weight after PNGase F digestion suggested that N-glycans were attached to both arms of $\mathrm{F}(\mathrm{ab}){ }_{2}$ fraction symmetrically. 
fibril proteins [6, 14, 42-44]. While the function of IgG Fab glycosylation has not been fully evaluated, its influence on antigen binding has been well established with monoclonal antibodies [7-9, 45]. Only a few associations of glycoforms of IgG Fab fragments with diseases (e.g. ANCA-associated systemic vasculitis) and with pregnancy have been reported $[4,46]$. Recently, a series of high-throughput, sensitive methods have been established to effectively analyze IgG Fab glycans with mass spectrometry [46] or capillary electrophoresis $[47,48]$. These methods have become powerful tools for IgG Fab glycosylation analysis, however, they cannot successfully fractionate whole Fab glycosylated IgG molecules from IgG mixtures. The present study provides an effective method to fractionate Fab glycosylated IgG, that could accurately distinguish Fab asymmetrically glycosylated IgG and Fab symmetrically glycosylated IgG with significant scientific and clinical implications.

\section{MATERIALS AND METHODS}

\section{Ethics statement}

The Ethical Committee of Shantou University Medical College has approved all experiments. All donors have provided written informed consent.

\section{Isolation of IgG from human serum}

Human serum was obtained through centrifuging healthy adult donors' peripheral blood at $500 \times \mathrm{g}$ for 5 minutes at $4{ }^{\circ} \mathrm{C}$. Total $\mathrm{IgG}$ was purified from serum using affinity chromatography with Recombinant Protein G Agarose according to the manufacturer's instructions (Invitrogen, California, USA).

\section{ConA affinity chromatography}

Human IgG was fractionated with lectin affinity chromatography using ConA Sepharose 4B following the manufactuer's description (GE Healthcare, Connecticut, USA). In brief, IgG was diluted with Binding Buffer (20 mM Tris-Hcl pH 7.4 containing $0.5 \mathrm{M} \mathrm{NaCl}, 1 \mathrm{mM}$ $\mathrm{CaCl}_{2}, 1 \mathrm{mM} \mathrm{MgCl}$, and $1 \mathrm{mM} \mathrm{MnCl}_{2}$ ) and loaded onto $2 \mathrm{~mL}$ of Sepharose 4B-linked ConA column. The column was further washed with Binding Buffer to remove the unbound $\mathrm{IgG}$, and all these unbound fractions were mixed in order to obtain the unbound IgG named ConA- IgG. The fraction bound to the ConA column was orderly eluted with Elution Buffer (25 mM Tris-Hcl pH 7.4 containing $0.2 \mathrm{M} \mathrm{NaCl}$ and $0.15 \mathrm{M} \alpha$-D-methylmannoside) and Regeneration Buffer $\mathrm{pH}$ 4.5. The fraction isolated by elution with Elution Buffer was named as ConA+ IgG, and the fraction isolated by elution with Regeneration Buffer $\mathrm{pH} 4.5$ was named as ConA++ IgG. The affinity chromatography of denatured IgG, Fab fragment, Fc fragment and $\mathrm{F}(\mathrm{ab})_{2}$, fragment were performed identically to IgG. The concentration of all fractions were performed with Amicon Ultra-4 Cnetrifugal Filter Units, 10kDa (Millipore, Massachusetts, USA) and the concentrations were determined with absorbance at $\mathrm{A}_{280}$.

\section{Production of IgG fragments}

$\mathrm{Fab}$ and Fc fragments were produced from IgG using Pierce Fab Preparation kit and F(ab') ${ }_{2}$ fragment were prepared with Pierce $\left.\mathrm{F}(\mathrm{ab})_{2}\right)_{2}$ Preparation kit following the manufacturer's instructions (Pierce Biotechnology, Illinois, USA).

\section{Deglycosylation enzymatic treatment}

The deglycosylation enzymatic treatment of $\operatorname{IgG}$ and its Fab, Fc or F(ab'), fragments was performed according to manufacturer's instructions of PNGase F (NEB, Massachusetts, USA).

\section{SDS PAGE}

The IgG and its Fab, Fc or F(ab') 2 fragments with or without PNGase F treatment were separated with SDS PAGE using $10 \%$ polyacrylamide BisTris gels under non-reducing or reducing conditions. The gels were then stained with silver or transferred to nitrocellulose membranes (Whatman, Dassel, Germany) and then examined with Western blot or lectin stain as described below.

\section{Silver stain}

After SDS PAGE, the gels were fixed with fixation fluid (10\% acetic acid and 40\% ethanol) for 30 minutes to overnight. The gels were incubated with $30 \%$ ethanol containing $0.5 \mathrm{M}$ sodium acetate anhydrous and $0.02 \mathrm{M}$ sodium thiosulfate for 30 minutes. Staining was performed with staining buffer $(0.01 \mathrm{M}$ silver nitrate combining with $0.05 \%$ formaldehyde) for 40 minutes after washing three times in deionized water for 10 minutes each. The gels were developed with a solution containing $0.24 \mathrm{M}$ sodium carbonate anhydrous and $0.05 \%$ formaldehyde. The developing process was terminated with $5 \%$ acetic acid and the gels were preserved in deionized water.

\section{Western blot}

Protein aliquots were electrophoresed on SDS PAGE and then transferred to nitrocellulose filter membrane (Whatman, Dassel, Germany). The blots were blocked with 5\% skim milk in TBST (10 mM Tris Base, $150 \mathrm{mM} \mathrm{NaCl}, 0.1 \%$ Tween-20, $\mathrm{pH} \mathrm{8.0)} \mathrm{for} 1$ hour at room temperature, and incubated overnight with primary antibodies. Rabbit anti-human IgG $\gamma$ Fc region antibody (Dako, Copenhagen, Denmark), mouse anti-human Ig $\kappa$ chain antibody (ZSGB-BIO, Beijing, China) and mouse 
anti-human Ig $\lambda$ chain antibody (ZSGB-BIO, Beijing, China) at a dilution of $1: 1,000$ were used as primary antibodies. Goat anti-rabbit IgG-680 (1:10,000; LI-COR, Nebraska, USA) and goat anti-mouse IgG-680 (1:10,000; LI-COR, Nebraska, USA) were used as secondary antibodies. The blots were examined with a Odyssey imaging system (LI-COR, Nebraska, USA).

\section{Lectin stain}

For ConA stain, the membrane was blocked in Carbo-Free Blocking Solution (Vector labs, California, USA) for 1 hour and incubated with $2 \mu \mathrm{g} / \mathrm{ml}$ biotin labeled ConA (Vector labs, California, USA) for 1 hour. After washing with TPBS (0.01 M PBS, pH 7.4, 0.05\% Tween-20), incubated the membrane with VECTASTAIN ABC-AP kit (Vector labs, California, USA) for 30 minutes according to the kit instructions. BCIP/NBT substrate kit (Vector labs, California, USA) was used for development. All procedures were performed at room temperature.

For SNA (Sambucus nigra agglutinin) stain, experiment was performed using Dig Glycan Differentiation Kit following the manufacturer's instructions (Roche, Basel, Switzerland).

\section{Protein identification with mass spectrometry}

After silver stain of the SDS-PAGE gels, the object bands were cut out. Protein samples were identified with MALDI TOF MS (Matrix-Assisted Laser Desorption/ Ionization Time of Flight Mass Spectrometry) analysis.

\section{Abbreviations}

Asn 297, asparagine 297; ConA, Concanavalin A;Gal, galactose; GlcNAc, N-acetylglucosamine; IgG, Immunoglobulin G; Man, mannose.

\section{ACKNOWLEGMENTS}

We thank Chun Ruan, Zuoqing Su and Guizhou She for assistance with the experiments.

\section{CONFLICTS OF INTEREST}

The authors declare no financial or commercial conflicts of interest.

\section{FUNDING}

This work was supported by grants from National Natural Science Foundation of China (30971150).

\section{REFERENCES}

1. Jefferis R. Glycosylation of recombinant antibody therapeutics. Biotechnol Prog. 2005; 21:11-16.

2. Krapp S, Mimura Y, Jefferis R, Huber R, Sondermann P. Structural analysis of human IgG-Fc glycoforms reveals a correlation between glycosylation and structural integrity. J Mol Biol. 2003; 325:979-989.

3. Arnold JN, Wormald MR, Sim RB, Rudd PM, Dwek RA. The impact of glycosylation on the biological function and structure of human immunoglobulins. Annu Rev Immunol. 2007; 25:21-50.

4. Holland M, Yagi H, Takahashi N, Kato K, Savage CO, Goodall DM, Jefferis R. Differential glycosylation of polyclonal IgG, IgG-Fc and IgG-Fab isolated from the sera of patients with ANCA-associated systemic vasculitis. Biochim Biophys Acta. 2006; 1760:669-677.

5. Stadlmann J, Pabst M, Altmann F. Analytical and Functional Aspects of Antibody Sialylation. J Clin Immunol. 2010; 30:S15-9.

6. Sox HC, Jr, Hood L. Attachment of carbohydrate to the variable region of myeloma immunoglobulin light chains. Proc Natl Acad Sci U S A. 1970; 66:975-982.

7. Fujimura Y, Tachibana H, Eto N, Yamada K. Antigen binding of an ovomucoid-specific antibody is affected by a carbohydrate chain located on the light chain variable region. Biosci Biotechnol Biochem. 2000; 64:2298-2305.

8. Wright A, Tao MH, Kabat EA, Morrison SL. Antibody variable region glycosylation: position effects on antigen binding and carbohydrate structure. EMBO J. 1991; 10:2717-2723.

9. Coloma MJ, Trinh RK, Martinez AR, Morrison SL. Position effects of variable region carbohydrate on the affinity and in vivo behavior of an anti- $(1 \longrightarrow 6)$ dextran antibody. J Immunol. 1999; 162:2162-2170.

10. Youings A, Chang SC, Dwek RA, Scragg IG. Site-specific glycosylation of human immunoglobulin $\mathrm{G}$ is altered in four rheumatoid arthritis patients. Biochem J. 1996; 314:621-630.

11. Mimura Y, Ashton PR, Takahashi N, Harvey DJ, Jefferis R. Contrasting glycosylation profiles between Fab and Fc of a human IgG protein studied by electrospray ionization mass spectrometry. J Immunol Methods. 2007; 326:116-126.

12. Borel IM, Gentile T, Angelucci J, Margni RA, Binaghi RA. Asymmetrically glycosylated IgG isolated from nonimmune human sera. Biochim Biophys Acta. 1989; 990:162-164.

13. Kobata A, Endo T. Immobilized lectin columns: useful tools for the fractionation and structural analysis of oligosaccharides. J Chromatogr. 1992; 597:111-122.

14. Spiegelberg HL, Abel CA, Fishkin BG, Grey HM. Localization of the carbohydrate within the variable region of light and heavy chains of human gamma g myeloma proteins. Biochemistry. 1970; 9:4217-4223. 
15. Chen Z, Qiu X, Gu J. Immunoglobulin expression in nonlymphoid lineage and neoplastic cells. Am J Pathol. 2009; 174:1139-1148.

16. Wright A, Morrison SL. Antibody variable region glycosylation: biochemical and clinical effects. Springer Semin Immunopathol. 1993; 15:259-273.

17. Osawa T, Tsuji T. Fractionation and structural assessment of oligosaccharides and glycopeptides by use of immobilized lectins. Annu Rev Biochem. 1987; 56:21-42.

18. Shibuya N, Goldstein IJ, Broekaert WF, Nsimba-Lubaki M, Peeters B, Peumans WJ. The elderberry (Sambucus nigra L.) bark lectin recognizes the Neu5Ac(alpha 2-6)Gal/ GalNAc sequence. J Biol Chem. 1987; 262:1596-1601.

19. Wang WC, Cummings RD. The immobilized leukoagglutinin from the seeds of Maackia amurensis binds with high affinity to complex-type Asn-linked oligosaccharides containing terminal sialic acid-linked alpha-2,3 to penultimate galactose residues. J Biol Chem. 1988; 263:4576-4585.

20. Shibuya N, Goldstein IJ, Van Damme EJ, Peumans WJ. Binding properties of a mannose-specific lectin from the snowdrop (Galanthus nivalis) bulb. J Biol Chem. 1988; 263:728-734.

21. Crowley JF, Goldstein IJ, Arnarp J, Lonngren J. Carbohydrate binding studies on the lectin from Datura stramonium seeds. Arch Biochem Biophys. 1984; 231: 524-533.

22. Goldstein IJ, Hollerman CE, Smith EE. ProteinCarbohydrate Interaction. Ii. Inhibition Studies on the Interaction of Concanavalin a with Polysaccharides. Biochemistry. 1965; 4:876-883.

23. Poljak RJ. Three-dimensional structure, function and genetic control of immunoglobulins. Nature. 1975; 256:373-376.

24. Deisenhofer J. Crystallographic refinement and atomic models of a human Fc fragment and its complex with fragment B of protein A from Staphylococcus aureus at 2.9and 2.8-A resolution. Biochemistry. 1981; 20:2361-2370.

25. Stadlmann J, Weber A, Pabst M, Anderle H, Kunert R, Ehrlich HJ, Peter Schwarz H, Altmann F. A close look at human IgG sialylation and subclass distribution after lectin fractionation. Proteomics. 2009; 9:4143-4153.

26. Scallon BJ, Tam SH, McCarthy SG, Cai AN, Raju TS. Higher levels of sialylated Fc glycans in immunoglobulin $\mathrm{G}$ molecules can adversely impact functionality. Mol Immunol. 2007; 44:1524-1534.

27. Anthony RM, Nimmerjahn F, Ashline DJ, Reinhold VN, Paulson JC, Ravetch JV. Recapitulation of IVIG antiinflammatory activity with a recombinant IgG Fc. Science. 2008; 320:373-376.

28. Kaneko Y, Nimmerjahn F, Ravetch JV. Anti-inflammatory activity of immunoglobulin $\mathrm{G}$ resulting from Fc sialylation. Science. 2006; 313:670-673.

29. Natsume A, Wakitani M, Yamane-Ohnuki N, ShojiHosaka E, Niwa R, Uchida K, Satoh M, Shitara K. Fucose removal from complex-type oligosaccharide enhances the antibody-dependent cellular cytotoxicity of single-geneencoded antibody comprising a single-chain antibody linked the antibody constant region. J Immunol Methods. 2005; 306:93-103.

30. Shinkawa T, Nakamura K, Yamane N, Shoji-Hosaka E, Kanda Y, Sakurada M, Uchida K, Anazawa H, Satoh M, Yamasaki M, Hanai N, Shitara K. The absence of fucose but not the presence of galactose or bisecting $\mathrm{N}$-acetylglucosamine of human IgG1 complex-type oligosaccharides shows the critical role of enhancing antibody-dependent cellular cytotoxicity. J Biol Chem. 2003; 278:3466-3473.

31. Takahashi M, Kuroki Y, Ohtsubo K, Taniguchi N. Core fucose and bisecting GlcNAc, the direct modifiers of the N-glycan core: their functions and target proteins. Carbohydr Res. 2009; 344:1387-1390.

32. Masuda K, Kubota T, Kaneko E, Iida S, Wakitani M, KobayashiNatsume Y, Kubota A, Shitara K, Nakamura K. Enhanced binding affinity for FcgammaRIIIa of fucose-negative antibody is sufficient to induce maximal antibody-dependent cellular cytotoxicity. Mol Immunol. 2007; 44:3122-3131.

33. Malhotra R, Wormald MR, Rudd PM, Fischer PB, Dwek RA, Sim RB. Glycosylation changes of IgG associated with rheumatoid arthritis can activate complement via the mannose-binding protein. Nat Med. 1995; 1:237-243.

34. Yuan S, Li Q, Zhang Y, Huang C, Wu H, Li Y, Liu Y, Yu N, Zhang H, Lu G, Gao Y, Gao Y, Guo X. Changes in antithyroglobulin IgG glycosylation patterns in Hashimoto's thyroiditis patients. J Clin Endocrinol Metab. 2015; 100:717-724.

35. Parekh RB, Dwek RA, Sutton BJ, Fernandes DL, Leung A, Stanworth D, Rademacher TW, Mizuochi T, Taniguchi T, Matsuta K, et al. Association of rheumatoid arthritis and primary osteoarthritis with changes in the glycosylation pattern of total serum IgG. Nature. 1985; 316:452-457.

36. Bondt A, Selman MH, Deelder AM, Hazes JM, Willemsen SP, Wuhrer M, Dolhain RJ. Association between galactosylation of immunoglobulin $\mathrm{G}$ and improvement of rheumatoid arthritis during pregnancy is independent of sialylation. J Proteome Res. 2013; 12:4522-4531.

37. Parekh R, Roitt I, Isenberg D, Dwek R, Rademacher T. Agerelated galactosylation of the $\mathrm{N}$-linked oligosaccharides of human serum IgG. J Exp Med. 1988; 167:1731-1736.

38. Ruhaak LR, Uh HW, Beekman M, Koeleman CA, Hokke CH, Westendorp RG, Wuhrer M, HouwingDuistermaat JJ, Slagboom PE, Deelder AM. Decreased levels of bisecting GlcNAc glycoforms of IgG are associated with human longevity. PLoS One. 2010; 5:e12566.

39. Yamada E, Tsukamoto Y, Sasaki R, Yagyu K, Takahashi N. Structural changes of immunoglobulin G oligosaccharides with age in healthy human serum. Glycoconj J. 1997; 14:401-405.

40. Kasermann F, Boerema DJ, Ruegsegger M, Hofmann A, Wymann S, Zuercher AW, Miescher S. Analysis and 
functional consequences of increased Fab-sialylation of intravenous immunoglobulin (IVIG) after lectin fractionation. PLoS One. 2012; 7:e37243.

41. Rademacher TW, Parekh RB, Dwek RA. Glycobiology. Annu Rev Biochem. 1988; 57:785-838.

42. Fykse EM, Sletten K, Husby G, Cornwell GG, 3rd. The primary structure of the variable region of an immunoglobin IV light-chain amyloid-fibril protein (AL GIL). The Biochem J. 1988; 256:973-980.

43. Holm E, Sletten K, Husby G. Structural studies of a carbohydrate-containing immunoglobulin-lambda-lightchain amyloid-fibril protein (AL) of variable subgroup III. Biochem J. 1986; 239:545-551.

44. Tveteraas T, Sletten K, Westermark P. The amino acid sequence of a carbohydrate-containing immunoglobulinlight-chain-type amyloid-fibril protein. Biochem J. 1985; 232:183-190.

45. Gala FA, Morrison SL. V region carbohydrate and antibody expression. J Immunol. 2004; 172:5489-5494.
46. Bondt A, Rombouts Y, Selman MH, Hensbergen PJ, Reiding KR, Hazes JM, Dolhain RJ, Wuhrer M. Immunoglobulin $\mathrm{G}$ (IgG) Fab glycosylation analysis using a new mass spectrometric high-throughput profiling method reveals pregnancy-associated changes. Mol Cell Proteomics. 2014; 13:3029-3039.

47. Mahan AE, Tedesco J, Dionne K, Baruah K, Cheng HD, De Jager PL, Barouch DH, Suscovich T, Ackerman M, Crispin M, Alter G. A method for high-throughput, sensitive analysis of IgG Fc and Fab glycosylation by capillary electrophoresis. J Immunol Methods. 2015; 417:34-44.

48. Brown EP, Normandin E, Osei-Owusu NY, Mahan AE, Chan YN, Lai JI, Vaccari M, Rao M, Franchini G, Alter G, Ackerman ME. Microscale purification of antigen-specific antibodies. J Immunol Methods. 2015; 425:27-36. 